\title{
Symmetric Games With Only Asymmetric Equilibria*
}

\author{
Mark Fey ${ }^{\dagger}$ \\ September 2, 2011
}

\begin{abstract}
It is known that not every symmetric game has a symmetric equilibrium because there are examples of symmetric games that fail to have any equilibria at all. But this leads to the following question: If a symmetric game has a Nash equilibrium, does it have a symmetric Nash equilibrium? In this note, we show that the answer to this question is no by providing two examples of symmetric games that have only asymmetric equilibria.
\end{abstract}

*John Duggan deserves thanks for useful suggestions on this topic.

${ }^{\dagger}$ Department of Political Science and Wallis Institute of Political Economy, University of Rochester. email: mark.fey@rochester.edu 


\section{Introduction}

When do symmetric games possess symmetric Nash equilibria? One answer to this question was given in the classic paper of Nash (1951), who showed that every finite symmetric game has a symmetric Nash equilibrium. Expanding on this answer, several authors have shown that continuous symmetric games and certain kinds of discontinuous symmetric games also always have symmetric equilibria (Dasgupta and Maskin, 1986; Yang, 1994; Reny, 1999; Becker and Damianov, 2006). Of course, this answer only goes so far, as there are examples of symmetric games in which Nash equilibria fail to exist, e.g. Example 3 in Dasgupta and Maskin (1986). Thus, the existence of symmetric equilibria is guaranteed in some broad classes of symmetric games, but not in all such games.

But the examples of nonexistence of equilibria in symmetric games have neither symmetric nor asymmetric equilibria, which leads us to refine our opening question as follows: When do symmetric games with equilibria possess symmetric equilibria? Or put another way, is it true that if a symmetric game has a Nash equilibrium, then it has a symmetric Nash equilibrium?

If we restrict ourselves to pure strategy Nash equilibria, the answer to this question is obviously no, as demonstrated by the game of Chicken. The answer to this question remains negative in infinite games, as demonstrated recently by Amir, Garcia and Knauff (2010). They construct two general classes of infinite games that always possess only asymmetric pure strategy Nash equilibria. But these games, as with the game of Chicken, always possess symmetric mixed strategy Nash equilibria. So this existing work does not answer our question when considering both pure and mixed equilibria; while these games possess only asymmetric pure strategy equilibria, they do not possess only asymmetric equilibria when mixed strategies are allowed.

Therefore, in this paper we consider the set of pure and mixed equilibria in examining whether symmetric games with equilibria always possess 
symmetric equilibria. Indeed, we show that this statement is true for the class of two-player, zero-sum, symmetric games. If such a game has a Nash equilibrium, then it has a symmetric Nash equilibrium.

Does this result extend to symmetric games that are not zero-sum? We show that the answer to this question is no by providing two examples of symmetric games that have only asymmetric equilibria. The first example we provide is "close" to zero-sum in that it is zero-sum at all but two points in the unit square. On the other hand, the second example is a symmetric game in which the players share the same payoff function for all outcomes. Thus, these examples show that there are symmetric games with near complete conflict as well as complete agreement that possess only asymmetric equilibria.

\section{Symmetric Games}

Let $G=\left(S, S, u_{1}, u_{2}\right)$ be a two-player strategic form game in which $S$ is a Hausdorff space and $u_{1}, u_{2}: S \times S \rightarrow \mathbb{R}$ are Borel measurable and bounded. Throughout we assume that $G$ is symmetric, so that $u_{1}\left(s_{1}, s_{2}\right)=u_{2}\left(s_{2}, s_{1}\right)$ for all $s_{1}, s_{2} \in S$. A mixed strategy for player $i$, denoted $\sigma_{i}$, is an element of $\Sigma$, the set of Borel probability measures on $S$. The definition of the expected payoffs $u_{i}\left(\sigma_{1}, \sigma_{2}\right)$ generated by a pair of mixed strategies and the definition of Nash equilibrium are standard. ${ }^{1}$ A Nash equilibrium $\left(\sigma_{1}^{*}, \sigma_{2}^{*}\right)$ is symmetric if $\sigma_{1}^{*}=\sigma_{2}^{*}$. Finally, the game $G$ is zero-sum if $u_{1}\left(s_{1}, s_{2}\right)=-u_{2}\left(s_{1}, s_{2}\right)$ for all $s_{1}, s_{2} \in S$.

\section{Symmetric Equilibria in Symmetric Zero-Sum Games}

We begin by stating an easily proved result on two-player zero-sum symmetric games. The proof relies on the well-known fact that all equilibria in two-player zero-sum games are interchangeable, meaning that if $\left(\sigma_{1}^{*}, \sigma_{2}^{*}\right)$ and

\footnotetext{
${ }^{1}$ See, for example, Fudenberg and Tirole (1991).
} 
$\left(\sigma_{2}^{*}, \sigma_{1}^{*}\right)$ are Nash equilibria, then so is $\left(\sigma_{1}^{*}, \sigma_{1}^{*}\right) .^{2}$

Proposition 1. If a two-player zero-sum symmetric game has a Nash equilibrium, then it has a symmetric Nash equilibrium.

Proof. Suppose a two-player zero-sum symmetric game has a Nash equilibrium $\left(\sigma_{1}^{*}, \sigma_{2}^{*}\right)$. Then by symmetry, the strategy profile $\left(\sigma_{2}^{*}, \sigma_{1}^{*}\right)$ is also a Nash equilibrium. As all equilibria in zero-sum games are interchangeable, it follows that $\left(\sigma_{1}^{*}, \sigma_{1}^{*}\right)$ is a Nash equilibrium.

It should be emphasized that this result holds for all two-player zero-sum symmetric games; no additional topological assumptions about the strategy sets or continuity assumptions about the utility functions are required. The simple proof given above relies on the interchangeability of equilibrium strategies and this is the only way in which the zero-sum condition enters. Thus, this proposition holds for any class of two-player symmetric games in which equilibrium strategies are interchangeable. As a consequence, the result of Proposition 1 holds for all two-player symmetric strictly competitive games (Friedman, 1983) and, even more broadly, for all two-player symmetric weakly unilaterally competitive games (Kats and Thisse, 1992).

\section{Symmetric and Asymmetric Equilibria in Symmet- ric Games}

We now show that Proposition 1 does not hold in all two-player symmetric games. We do so by providing two examples of symmetric games which possess only asymmetric equilibria.

Our first example is the following two-player symmetric game $G_{1}$, which is based on an example of Ville (1938) and discussed by Dresher (1961). The

\footnotetext{
${ }^{2}$ See, for example, Luce and Raiffa (1957, p.66).
} 


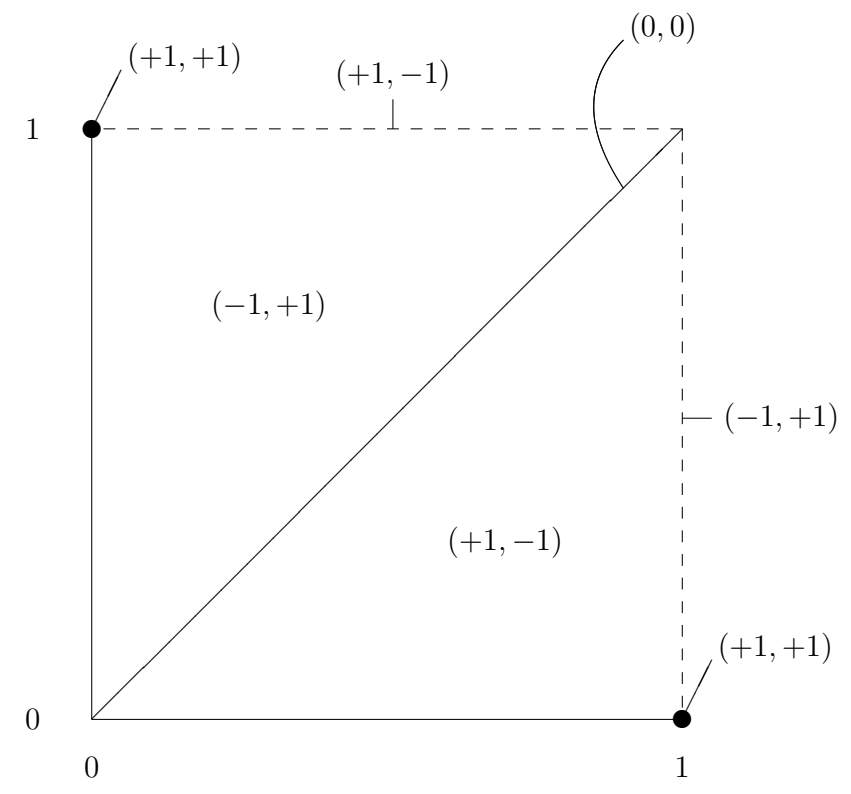

Figure 1: The game $G_{1}$ : a symmetric game with only asymmetric equilibria game $G_{1}$ is given by the strategy set $S=[0,1]$ and payoffs defined as

$$
u\left(s_{i}, s_{j}\right)= \begin{cases}(0,0) & \text { if } s_{i}=s_{j} \\ (+1,-1) & \text { if } s_{j}<s_{i}<1 \text { or } 0<s_{i}<s_{j}=1 \\ (-1,+1) & \text { if } s_{i}<s_{j}<1 \text { or } 0<s_{j}<s_{i}=1 \\ (+1,+1) & \text { if } s=(1,0) \text { or } s=(0,1)\end{cases}
$$

This game is pictured in Figure 1. As can be seen in this figure, this game is "almost" zero-sum in that it is zero-sum everywhere except for the two points $(0,1)$ and $(1,0)$. This figure also makes clear that these two points are both asymmetric Nash equilibria, as both players are receiving their maximum possible payoff.

We now show that this game does not have a symmetric Nash equilibrium. For a proof by contradiction, suppose $(\sigma, \sigma)$ is a symmetric Nash equilibrium. We consider two cases. First, suppose that $\sigma(\{1\})>0$, i.e., the symmetric 
equilibrium strategy has a mass point at $x=1$. This means that $x=1$ must be a pure strategy best response to $\sigma$, but the expected payoff of choosing $x=1$ is strictly less than the expected payoff of choosing some $z$ arbitrarily close to one. Therefore $\sigma$ cannot have a mass point at $x=1$.

For the second case, suppose that $\sigma(\{1\})=0$. This implies that with probability one the equilibrium outcomes are zero-sum. Therefore the equilibrium payoff of $(\sigma, \sigma)$ to both players is equal to zero. But by choosing some $z$ arbitrarily close to one a player can achieve a positive expected payoff, which is a profitable deviation. This rules out the second case and establishes that this game does not have a symmetric Nash equilibrium.

As mentioned above, there are only two points in the unit square at which this game fails to be zero-sum and yet the conclusion of Proposition 1 does not hold. So this example shows that only a small departure from zero-sum is enough to preclude the existence of symmetric equilibria in symmetric games with equilibria.

Because the game $G_{1}$ is "almost" zero-sum, it reflects a situation of almost complete conflict. ${ }^{3}$ But what about the opposite situation, in which players are in complete agreement, instead of conflict? In our second example, we give a symmetric game in which the two players have identical preferences over all outcomes but which possesses only asymmetric equilibria. ${ }^{4}$

Consider the following two-player symmetric game $G_{2}$ with $S=[0,1]$ and payoffs given by

$$
u_{i}\left(s_{1}, s_{2}\right)= \begin{cases}\max \left\{s_{1}, s_{2}\right\} & \text { if } s \neq(1,1) \\ (0,0) & \text { if } s=(1,1)\end{cases}
$$

This game clearly has a continuum of equilibria. The (pure strategy)

\footnotetext{
${ }^{3}$ However, it is worth noting that $G_{1}$ is also a game of common interest (Aumann and Sorin, 1989) in that there are two strategy profiles which give both players their highest possible payoff.

${ }^{4}$ This example is related to an example of Dasgupta and Maskin (1986).
} 
equilibrium set is given by $\left\{\left(s_{1}, s_{2}\right) \mid s_{1}=1\right.$ or $\left.s_{2}=1\right\} \backslash(1,1)$.

We now show that this game does not have a symmetric equilibrium. For a proof by contradiction, suppose $(\sigma, \sigma)$ is a symmetric Nash equilibrium to $G_{2}$. If $\sigma(\{1\})>0$, then $x=1$ must be a pure strategy best response to $\sigma$. But $\sigma(\{1\})>0$ implies that the expected payoff from $x=1$ is strictly less than one, which is strictly less than the payoff of choosing some $z$ arbitrarily close to one. Thus $x=1$ is not a pure strategy best response to $\sigma$, a contradiction. On the other hand, if $\sigma(\{1\})=0$, then the expected payoff of $x=1$ is equal to one, which is strictly higher than the payoff of any $z<1$. Thus, choosing $x=1$ is strictly optimal in this case, contradicting the assumption that $\sigma(\{1\})=0$. This shows that $G_{2}$ has no symmetric Nash equilibria.

It is worth noting that the identical payoff of the players is discontinuous at only one point, namely the point $(1,1)$. Thus while continuity of payoffs ensures the existence of a symmetric equilibria, a single discontinuity point is enough to allow a game to have only asymmetric equilibria.

\section{Conclusion}

Although it is true that a two-player symmetric zero-sum game has a Nash equilibrium if and only if it has a symmetric Nash equilibrium, we have shown that this statement does not hold for all symmetric games. Indeed, our two examples illustrate that symmetric games can possess only asymmetric equilibria in cases of close to complete conflict over outcomes as well as complete agreement over outcomes.

Finally, it is useful to mention a couple of additional results that follow from our examples of symmetric games that possess only asymmetric equilibria. First, this conclusion also holds for other, weaker equilibria concepts such as $\varepsilon$-equilibrium. Both of the games described here have only asymmetric $\varepsilon$-equilibria. Second, it is easy to give a symmetric $n$ player version of the game $G_{2}$ which has only asymmetric equilibria. Specifically, suppose 
every player's utility is given by $\max \left(s_{1}, \ldots, s_{n}\right)$ for all strategy profiles except $(1, \ldots, 1)$, which is assigned utility zero. Then the profile $(1,0, \ldots, 0)$ is an asymmetric Nash equilibrium, but the argument given above for game $G_{2}$ shows this game does not have a symmetric Nash equilibrium. Thus, our basic finding holds for games with more than two players, as well. 


\section{References}

Amir, Rabah, Filomena Garcia and Malgorzata Knauff. 2010. "Symmetrybreaking in two-player games via strategic substitutes and diagonal nonconcavity." Journal of Economic Theory 145(5):1968-1986.

Aumann, Robert J. and Sylvain Sorin. 1989. "Cooperation and bounded recall." Games and Economic Behavior 1(1):5-39.

Becker, Johannes Gerd and Damian S. Damianov. 2006. "On the existence of symmetric mixed strategy equilibria." Economics Letters 90(1):84-87.

Dasgupta, Partha and Eric Maskin. 1986. "The existence of equilibrium in discontinuous economic games, I: Theory." The Review of Economic Studies 53(1):1-26.

Dresher, Melvin. 1961. Games of strategy: theory and applications. Santa Monica, CA: Rand Corporation.

Friedman, James W. 1983. "On characterizing equilibrium points in two person strictly competitive games." International Journal of Game Theory 12(4):245-247.

Fudenberg, Drew and Jean Tirole. 1991. Game theory. MIT Press.

Kats, Amoz and Jacques François Thisse. 1992. "Unilaterally competitive games." International Journal of Game Theory 21(3):291-299.

Luce, R. Duncan and Howard Raiffa. 1957. "Games and decisions. Introduction and critical survey.".

Nash, John. 1951. "Non-cooperative games." Annals of mathematics 54(2):286-295.

Reny, Philip J. 1999. "On the existence of pure and mixed strategy Nash equilibria in discontinuous games." Econometrica 67(5):1029-1056. 
Ville, J. 1938. "Sur la théorie générale des jeux où intervient l'habilité des joueurs." Traite du calcul des probabilité et de ses applications 2(5).

Yang, Chun-Lei. 1994. "A simple extension of the Dasgupta-Maskin existence theorem for discontinuous games with an application to the theory of rentseeking." Economics Letters 45(2):181-183. 